\title{
Alternativas para um produto cárneo mais saudável: uma revisão
}

\author{
Alternatives for a healthier meat product: a review
}

\section{Autores | Authors}

凶 Débora Francielly de OLIVEIRA

Universidade Tecnológica Federal do Paraná (UTFPR)

Departamento Tecnologia de Alimentos

Av. dos Pioneiros, 3131 CEP: 86036-370

Londrina/PR - Brasil

e-mail: deborafolhe@hotmail.com

Alexandre Rodrigo COELHO Vânia de Cássia da Fonseca

BURGARDT

Elisabete Hiromi HASHIMOTO

Alessandra Machado LUNKES

João Francisco MARCHI

Ivane Benedetti TONIAL

Universidade Tecnológica Federal do

Paraná (UTFPR)

Departamento Tecnologia de Alimentos

e-mail: arcoelho@utfpr.edu.br vaniafonseca@utfpr.edu.br

elisabete@utfpr.edu.br amachado@utfpr.edu.br joaomarchi@utfpr.edu.br ivane@utfpr.edu.br

\ Autor Correspondente / Corresponding Author

Recebido / Received: 25/07/2013 Aprovado / Approved: 04/09/2013 Publicado / Published: set./2013

\section{Resumo}

Considerando-se a evidente busca por alimentos de rápido e fácil preparo, as comunidades industrial e científica vêm investindo no desenvolvimento de novos produtos que, além de atenderem a essa demanda, possam oferecer benefícios à saúde do consumidor. Dentre os alimentos que não demandam muito tempo para o preparo no domicílio, o hambúrguer merece destaque, em função do seu elevado consumo. No entanto, em razão de conter gordura saturada e por ser submetido a processo de fritura, o consumo demasiado desse produto pode ser prejudicial à saúde humana, podendo causar doenças crônicas, entre as quais, a obesidade e a hipertensão. Estudos têm demonstrado a possibilidade de substituição de ingredientes na formulação de hambúrgueres, com a intenção de incorporar substâncias com propriedades funcionais; portanto, substâncias que possam contribuir para a saúde e o bem-estar dos consumidores. Nesse contexto, esta revisão aborda questões referentes ao hambúrguer, no sentido de conceituar o produto e, à luz da literatura, oferecer alternativas possíveis e que têm sido estudadas para tornar esse alimento mais saudável, com foco na substituição de gordura animal por fibras e na redução do teor de cloreto de sódio.

Palavras-chave: Hambúrguer; Alimento funcional; Ômega-3; Fibras; Cloreto de potássio.

\section{Summary}

Considering the evident search for quick and easy food preparation, industrial and scientific communities have been investing in developing new products that fulfill this demand and may offer health benefits to consumer. Among foods that do not require much time for preparation at home, the burger deserves attention due to its high consumption. However, due to its saturated fat contents and being subjected to frying process, the excessive consumption of this product may be harmful to human health and may cause chronic diseases, including obesity and hypertension. Studies have demonstrated the possibility of ingredients substitution in the formulation intending to incorporate substances with functional properties, so that they can contribute to consumers' health and well-being. In this context, this paper describes issues related to the burger, to conceptualize the product and, in the light of the literature, to offer studied alternatives to make it healthier, focusing on replacing animal fat by fibers and on sodium chloride content reduction.

Key words: Burger; Functional food; Omega-3; Fiber; Potassium chloride. 


\section{Introdução}

Em função da aceleração do ritmo urbano, dado pelo tempo produtivista, é cada vez mais evidente a busca por alimentos industrializados de fácil preparo. No entanto, junto à escassez de tempo e à procura por alimentos de rápido e fácil preparo, vem a preocupação da população com os problemas de saúde que estes alimentos podem ocasionar.

Essa demanda tem motivado as comunidades industrial e científica a unirem esforços no sentido de ofertar alimentos industrializados que, além de nutrir, promovam o bem-estar e atuem como redutores dos riscos de doenças crônicas. Nesse contexto, surgiu a concepção de alimentos funcionais, aqueles que apresentam em sua composição componente(s) que oferece $(m)$ benefícios fisiológicos e bioquímicos, e não somente nutricionais.

A variedade de produtos cárneos que não demandam muito tempo para o preparo, disponibilizada nas gôndolas de supermercados, tornou-se um atrativo para os consumidores, contribuindo para que salsicha, salame, mortadela, linguiça, empanado, almôndegas e hambúrguer sejam opção crescente para o lanche de muitas famílias no mundo todo.

Em decorrência da sua praticidade de preparo e por possuir nutrientes que alimentam e saciam a fome rapidamente, o hambúrguer se tornou um produto consumido por todas as classes populares. No entanto, o consumo demasiado deste tipo de produto pode ser prejudicial à saúde humana, podendo ocasionar aumento da pressão arterial, excesso de gordura no sangue e obesidade, doenças estas, tidas como um problema de saúde pública e que, em tempos recentes, têm acometido além de adultos e idosos, crianças.

Diante do exposto, o trabalho propõe, baseado na literatura, alternativas para formulações de hambúrgueres de carne bovina mais saudáveis através da substituição de cloreto de sódio por outros sais ou acréscimo de ingredientes com propriedades funcionais, os quais, por apresentarem em sua constituição substâncias bioativas, podem trazer benefícios à saúde e ao bem-estar dos consumidores.

Para essa abordagem teórica, realizaram-se buscas textuais na literatura científica, eletronicamente nas plataformas Portal de Periódicos Capes (CAPES), Scientifc Eletronic Base Library Online (SciELO), Web of Knowledge - banco de dados do Institute for Scientific Information (ISI), Science Direct, Google Acadêmico (GOOGLE), bem como nos sites de patentes LatipatEspacenet (Patentes latinoamericanas), PatentesOnline. es e Freepatentsonline (Patentes internacionais) (JENSEN et al., 2008), além de sites independentes, institucionais e governamentais, e outros ligados à regulamentação da produção de alimentos funcionais. A literatura impressa, como revistas e livros com assuntos inerentes ao discutido e em áreas correlatas, também foi consultada. Entre as palavras-chave utilizadas na busca, citam-se: alimentos funcionais; hambúrguer; carne bovina; substitutos de gordura; cloreto de sódio; fibras; ácidos graxos, e doenças crônicas e degenerativas. Os critérios de inclusão para os estudos encontrados foram abordagens nutricional e de saúde, bem como as aplicações industriais de componentes bioativos e a substituição parcial do conteúdo de cloreto de sódio em produtos cárneos, com ênfase em hambúrgueres.

\section{Referencial teórico}

\subsection{Alimentação e saúde}

De acordo com o Brillat-Savarin (1825) apud Guia Alimentar para a População Brasileira (BRASIL, 2006), "o destino das nações depende daquilo e de como as pessoas se alimentam". Essa afirmação, que remonta há quase dois séculos, já demonstrava a relação vital entre a alimentação e a saúde.

A alimentação constitui uma das atividades humanas mais antigas e importantes, não só por razões biológicas evidentes, mas também por envolver aspectos econômicos, sociais, científicos, políticos, psicológicos e culturais, sendo todos fundamentais na dinâmica da evolução das sociedades (PROENÇA, 2010).

Entretanto, a sociedade moderna tem levado a hábitos alimentares que afetam de maneira significativa a saúde dos consumidores, podendo contribuir negativamente para a qualidade de vida das pessoas (KARANJA et al., 2007). A mudança dos hábitos alimentares relatados por Karanja et al. (2007) pode ser decorrente da aceleração do ritmo urbano e da maior inserção da mulher no mercado de trabalho, o que ocasiona escassez de tempo para preparar alimentos no domicílio; por isso, é cada vez mais evidente a busca por alimentos que não demandam muito tempo para o preparo e que, muitas vezes, podem não ser saudáveis, podendo levar a sérios problemas de saúde.

\subsection{Doenças provenientes de alimentação inadequada}

De acordo com Giuntini et al. (2006), uma alimentação baseada em alimentos ricos em gorduras, açúcares e sódio, e pobre em vitaminas, sais minerais, proteínas e fibras pode resultar no processo de doença. No entanto, vale comentar que, para a manutenção das funções vitais do organismo humano, recomenda-se uma dieta diária que possa fornecer 2.000 quilocalorias, conseguidas somente com a ingestão de macronutrientes, como os carboidratos e lipídios, tidos também como 
nutrientes energéticos por fornecer energia de $4 \mathrm{kcal} / \mathrm{g}$ e $9 \mathrm{kcal} / \mathrm{g}$, respectivamente. Assim, uma dieta mais indicada é aquela equilibrada, ou seja, com ingestão de alimentos ricos em proteínas, fibras e micronutrientes (sais minerais e vitaminas), com teores reduzidos de sódio, porém que possa fornecer energia. Portanto, uma dieta que apresente também consideráveis níveis lipídicos e de açúcares, desde que, é claro, esteja associada com exercícios físicos.

Dentre as doenças provenientes de alimentação inadequada, citam-se: hipertensão (aumento da pressão arterial), obesidade (excesso de peso), diabetes (taxa de glicose elevada no sangue) e dislipidemia (excesso de gordura no sangue) (COXSON et al., 2013).

\subsection{Hipertensão}

Freitas et al. (2001) relataram que a hipertensão arterial - considerada um problema de saúde pública por sua magnitude, risco e dificuldades de controle, bem como por ser reconhecida como um dos mais importantes fatores de risco para o desenvolvimento de acidente vascular cerebral e infarto do miocárdio - está, muitas vezes, associada ao elevado consumo de sódio e ao excesso de peso. O resultado desse estudo apontou que a prevalência de hipertensão sistêmica foi maior em indivíduos com histórico de hipertensão na família, seguidos de portadores de diabetes.

Sichieri et al. (2000), quando fizeram uma abordagem teórica sobre recomendações de alimentação saudável para a população brasileira, concluíram que "[...] comer pouco sal" é uma das mensagens principais para a saúde das pessoas. Phillips (2003), com trabalho intitulado 'Don't pass the salt', ressaltou a importância para a saúde de não exagerar no conteúdo de sal. Não obstante, Coxson et al. (2013), sob três diferentes premissas de modelagem computacional, corroboraram, recentemente, com resultados obtidos, os quais demonstraram os efeitos benéficos para redução dos níveis de mortalidade em decorrência de hipertensão, através da diminuição do consumo de sódio. No mesmo sentido, Busch et al. (2013) contribuíram com uma revisão sobre a percepção da redução de sódio na dieta dos consumidores de alimentos industrializados, os quais, segundo os autores, na grande maioria, apresentam elevados teores de cloreto de sódio.

Essa preocupação com o excesso de sódio na dieta tem levado pesquisadores do mundo todo a unir esforços no sentido de oferecer alimentos com teor reduzido desse constituinte. Uma opção crescente para a substituição de cloreto de sódio $(\mathrm{NaCl})$ em produtos alimentícios é a sua permuta por cloreto de potássio (KCl). Dentre os vários experimentos realizados com a intenção de reduzir o sal de produtos cárneos, Paulino et al. (2006), Nascimento et al. (2007) e Ciriano et al. (2013) reduziram o percentual de $\mathrm{NaCl}$ em embutidos cárneos suínos, salsichas e salames, respectivamente, através da substituição parcial desse constituinte por $\mathrm{KCl}$; foi observado que a substituição de $25,0 \%$ de $\mathrm{NaCl}$ não interferiu significativamente na capacidade de retenção de água, no sabor salgado e na estabilidade da emulsão das salsichas formuladas (NASCIMENTO et al., 2007). Na China, em estudo realizado com o objetivo de verificar a aceitação de alimentos com substituição parcial de $\mathrm{NaCl}$ por $\mathrm{KCl}$, Li et al. (2009) obtiveram resultados também satisfatórios, no sentido de que a substituição não interferiu no sabor dos produtos avaliados, porém esses autores sugeriram uma nova avaliação com maior número de pessoas.

\subsubsection{Obesidade}

Assim como a hipertensão, a obesidade também é tida como um problema de saúde pública e representa o problema nutricional de maior ascensão entre a população observado nos últimos anos, sendo considerada uma epidemia mundial, presente tanto em países desenvolvidos como naqueles em desenvolvimento (MARIATH et al., 2007). Esta preocupação tem contribuído para o maior investimento por parte do Poder Público em programas que objetivam orientar e estimular a população a buscar uma alimentação mais saudável. Um estudo comprovou, concluindo que a ingestão de alimentos com altos teores de gorduras saturadas - como é o caso das gorduras encontradas em leites, carnes e seus derivados - é a principal causa do aumento de peso corporal (FRANCISCHI et al., 2000), desencadeando a obesidade.

\subsubsection{Colesterol}

De acordo com Mattes (1998), o aumento de colesterol na corrente sanguínea pode ocasionar entupimento de veias e artérias, causando o infarto e o derrame. $\mathrm{O}$ autor relatou ainda que o colesterol provém de duas fontes: no organismo (produzido pelo fígado) e decorrente da alimentação (ingestão de gorduras saturadas), o que implica dizer que o consumo em excesso de leites, carnes e seus derivados pode elevar os níveis de colesterol no sangue. Isso se justifica pelo fato de que os produtos de origem animal apresentam teores consideráveis de ácidos graxos saturados, sendo estes grandes vilões, por ocasionarem maior aumento dos níveis de colesterol na corrente sanguínea, quando comparados com os ácidos graxos poli-insaturados. Akoh (1998) corroborou tal relação quando sugeriu que o consumo de altas quantidades de gorduras aumenta o risco de obesidade, podendo ocasionar alguns tipos de câncer, sendo a ingestão de gorduras saturadas associada ao aumento do colesterol sanguíneo e às doenças coronarianas. Pinheiro e Penna (2004) 
complementaram ao sugerir a utilização de substitutos de gordura como boa opção de redução de gorduras saturadas em alimentos. O aumento das contribuições científicas que tratam da redução parcial ou total de gordura saturada, demonstrado na literatura, é prova de que tais substituições podem contribuir para a melhor saúde do consumidor.

\subsubsection{Diabetes}

Segundo Cercato et al. (2004), a falta ou a produção insuficiente de insulina, ou ainda a ação insuficiente da mesma, provoca aumento na taxa de glicose no sangue, ocasionando diabetes, doença caracterizada como outro problema de saúde pública. Rezende et al. (2007) acrescentaram que essa doença pode estar relacionada com o excesso de peso e a obesidade, estes, também relacionados com hábitos inadequados de alimentação.

Nesse contexto, o consumidor, preocupado com a saúde, tem cada vez mais buscado uma dieta saudável (SOUZA et al., 2011), aquela que, além de nutrir, promova redução do risco de diversas doenças e a manutenção do bem-estar físico e mental. Essa preocupação com a alimentação (MORAES e COLLA, 2006) tomou maiores proporções há aproximadamente 40 anos, com a concepção de alimentos saudáveis, os chamados alimentos funcionais. Em decorrência desta nova demanda por produtos de fácil e rápido preparo e que, ao mesmo tempo, possam ser saudáveis, as comunidades industrial e científica têm buscado alternativas adaptadas a essas condições urbanas (BARBOSA, 2001).

\subsection{Alimentos funcionais}

\subsubsection{Conceito}

A Portaria n. ${ }^{\circ} 398$ de 30 de abril de 1999, da Secretaria de Vigilância Sanitária do Ministério da Saúde do Brasil, define como funcional "Todo aquele alimento ou ingrediente que, além das funções nutricionais básicas, quando consumido como parte da dieta usual, produz efeitos metabólicos e/ou fisiológicos e/ou efeitos benéficos à saúde, devendo ser seguro para consumo sem supervisão médica" (BRASIL, 1999).

\subsubsection{Demais definições e considerações}

O termo "alimentos funcionais" apresenta diferentes definições, de acordo com o Órgão de Regulamentação ou o país de origem. Segundo Moraes e Colla (2006), a exemplo disso, no Japão, primeiramente introduzido, o termo foi definido em 1991 como "alimentos para uso específico de saúde" (Foods for Specified Health Use-FOSHU), enquanto que, no Reino Unido, o Ministério da Agricultura, Pesca e Alimentos (MAFF) (MORAES e COLLA, 2006) definiu alimentos funcionais como "alimentos cujo componente incorporado oferece benefício fisiológico e não apenas nutricional". Por outro lado, para os americanos, os termos "alimentos funcionais" e "nutracêuticos" têm sido usados conforme a definição estabelecida, e a dificuldade se encontra na regulamentação desses termos, pois deve haver uma diferenciação entre produtos que são vendidos e consumidos como alimentos (funcionais) e aqueles em que um componente, em particular, foi isolado, e é vendido na forma de barras, cápsulas e pós, entre outras apresentações (nutracêuticos) (BOCK et al., 2012).

Segundo Hasler (1998), no Brasil o termo é definido como "[...] qualquer alimento ou ingrediente que possa proporcionar um benefício à saúde, além dos nutrientes tradicionais que eles contêm [...]" (Comitê de Alimentos e Nutrição do Instituto de Medicina da Federação Náutica de Brasília).

Entretanto, em matéria de lei, um alimento funcional não tem nenhuma definição reconhecida pela FDC (Food, Drugs and Cosmetics). Diante disso e considerando a variação na definição do termo, a FDA (Food and Drug Administration) regula os alimentos funcionais baseada no uso que se pretende dar ao produto, na descrição presente nos rótulos ou nos ingredientes do produto. A partir desses critérios, a FDA classificou os alimentos funcionais em cinco categorias: alimento; suplementos alimentares; alimento para usos dietéticos especiais; alimento-medicamento, ou droga (NOONAN e NOONAN, 2004).

Em contrapartida, para vários países europeus, Estados Unidos e Brasil, alimento funcional pode ser considerado como alimento e não e/ou suplemento alimentar. Nessa perspectiva, para ambos os países, o alimento funcional deve apresentar primeiramente as funções nutricional e sensorial, sendo a funcionalidade a função terciária do alimento (KWAK e JUKES, 2001).

Apesar das diferenças de conceito existentes para o termo 'alimento funcional', é fato que a demanda por alimentos que proporcionem uma dieta saudável tem aumentado com os anos no mundo todo.

\subsection{Ingredientes alimentícios com propriedades bioativas}

São vários os ingredientes adicionados em produtos alimentícios com a intenção de conferir, aos mesmos, características funcionais. A crescente preocupação por uma alimentação saudável coloca alguns alimentos, como proteínas do soro de leite, soja, aveia, gergelim, chia, psyllium, quinoa e linhaça - esses dois últimos, com comprovação científica de suas propriedades funcionais - na lista de preferência de um número cada vez maior de consumidores (FREITAS e MORETTI, 2006; GUTKOSKI et al., 2007). Óleos como os de linhaça, oliva 
e soja (YUNES, 2010) e de canola (YOUSSEF e BARBUT, 2011) também vêm sendo adicionados em produtos cárneos, visando reduzir a quantidade de gordura animal nas formulações, gerando produtos com perfil de ácidos graxos e teores de colesterol mais adequados nutricionalmente em relação aos produtos tradicionais.

\subsubsection{Fibras alimentares}

Estudos revelaram resultados que comprovam a eficiência das fibras alimentares para o gerenciamento da função do intestino, com a intensificação de sua integridade e função absortiva, a manutenção da barreira intestinal e a normalização da microflora intestinal (BAXTER, 2001; CATALANI, 2003; RODRíGUEZ et al., 2006). De acordo com Mongeau et al. (1989) apud (ARAÚJO et al., 2009), as propriedades funcionais das fibras são determinadas pela inter-relação entre estruturas e características físico-químicas. Torres et al. (2006) acrescentaram que o conhecimento dessas propriedades funcionais das fibras pode ser explorado por profissionais da saúde e servir como base para recomendações dietéticas mais específicas.

Outros autores acrescentaram, ao relatarem que a adoção de uma dieta rica em fibras pode contribuir para diminuição das concentrações de lipídios séricos e dos níveis de adiposidade corporal e, ainda, baixar a incidência de mortes por isquemia do miocárdio, diabetes mellitus e certos tipos de câncer, além de uma maior expectativa de vida (SARTORELLI e FRANCO, 2003; FERREIRA et al., 2006; QI et al., 2006; RODRÍGUEZ et al., 2006). Em decorrência desses fatores, é cada vez mais evidente a utilização de fibras alimentares em diferentes farináceos e produtos lácteos; alguns exemplos: Okara, subproduto aquoso do extrato de soja em pães (BOWLES e DEMIATE, 2006), e granola e fruto-oligossacarídios em iorgurtes (SALES et al., 2008), bem como em produtos cárneos, atuando como substituto de gordura animal.

\subsubsection{Adição de fibras em produtos cárneos}

Com a industrialização da carne, surgiu uma alternativa para o aproveitamento dos cortes menos nobres, aumentando o lucro dos abatedouros. A diversificação da oferta inclui um grande número de produtos, como almôndegas, hambúrgueres, empanados, linguiças, mortadelas e salames, entre outros. Nos últimos anos, especial atenção tem sido dada aos alimentos com menor tempo de preparo, preço acessível, sabor agradável, boa qualidade e também com menor teor de gordura (COSTA, 2004).

Em razão de a gordura de origem animal estar relacionada a diversas doenças crônicas, pesquisadores têm somado esforços no sentido de estudar ingredientes que possam atuar como substitutos desse tipo de gordura em alimentos cárneos (SANTOS JÚNIOR et al., 2009).
De acordo com Bourscheid (2009), são três categorias de produtos que podem ser utilizadas como substitutos de gordura: proteínas não cárneas (proteína de soja e proteínas do leite), carboidratos bases (carragena, amidos, féculas e fibras) e misturas de ingredientes (junção de vários ingredientes que atuam na redução de gorduras); estes são rotineiramente empregados pela indústria de embutidos cárneos, principalmente por sua capacidade de formar géis aquosos, conferindo consistência ao produto final. Em contrapartida, segundo a American Dietetic Association (2005), o substituto de gordura ideal seria um composto seguro, que apresentasse todas as propriedades tecnológicas da gordura - melhor sabor e textura dos alimentos -, mas com baixo teor calórico; porém, esse composto não existe, embora estejam disponíveis no mercado diversos produtos com algumas dessas propriedades e que, em corretas combinação e proporção, permitem o desenvolvimento de grande número de produtos alimentícios (ADA, 2005).

Dentre as fibras recentemente utilizadas como ingredientes em alimentos cárneos industrializados, as adições de carragena, fibra solúvel de aveia ( $\beta$-glucana), farelo de aveia, fécula de mandioca, fibra de soja, fibra de ervilha, goma e farelo de linhaça (ANDERSON e BERRY, 2001; SEABRA et al., 2002; QUEIROZ et al., 2005; MARQUES, 2007; PIÑERO et al., 2008; MONEGO, 2009; CIRIANO et al., 2013) foram ressaltadas como alternativas para um produto mais saudável.

Dos produtos cárneos industrializados, nos quais tem sido estudada a influência da adição de fibras na diminuição do teor de gordura dos mesmos e da substituição parcial de cloreto de sódio, o hambúrguer tem recebido merecida atenção.

\subsection{Hambúrguer $\times$ Saúde}

De acordo com a legislação brasileira, hambúrguer é um 'produto cárneo industrializado, obtido de carne moída dos animais de açougue, adicionado ou não de tecido adiposo e ingredientes, moldado e submetido a processo tecnológico adequado. Trata-se de produto cru, semifrito, cozido, frito, congelado ou resfriado', conforme a sua classificação (BRASIL, 2000).

Segundo Colmenero (2000), produtos cárneos convencionais do tipo fast food possuem alto conteúdo de gordura, variando entre 20 e $30 \%$. Em contrapartida, Queiroz et al. (2005) discordaram ao dizer que a substituição de gordura animal pela utilização de miméticos (carboidratos, gomas e proteínas) pode proporcionar uma melhora nas características sensoriais desses produtos.

Vários experimentos sobre o uso de substitutos de gorduras em produtos cárneos, especialmente em 
hambúrguer, vêm sendo desenvolvidos, uma vez que, embora consumido por todas as classes populares em virtude da praticidade que representa e por possuir nutrientes que alimentam e saciam a fome rapidamente (HAUTRIVE et al., 2008; BARBOSA, 2010), o consumo demasiado desse tipo de produto pode ser prejudicial à saúde humana, causando obesidade e doenças, como hipertensão, diabettes mellitus e dislipidemias (ORTIGOZA, 2008).

Bragagnolo e Rodriguez-Amaya (2002), ao estudarem o conteúdo lipídico de diferentes cortes de carne suína, obtiveram resultados a partir dos quais constataram maior percentual de gordura saturada no toucinho $(38,0 \%)$, em relação aos valores observados para paleta $(36,0 \%)$ e pernil $(33,0 \%)$. Essa constatação implica sugerir a substituição parcial ou total do toucinho em formulações de hambúrguer e, com isso, tornar o produto mais saudável.

Costa (2004) corroborou esses dados relatando que, geralmente, as fibras são adicionadas em hambúrguer para reduzir o teor de ácidos graxos saturados dos mesmos, o que, segundo Bragagnolo e RodriguezAmaya (2002), confere características mais saudáveis ao produto.

\subsubsection{Redução de gorduras e de cloreto de sódio: alternativas para desenvolvimento de hambúrguer mais saudável}

São várias as alternativas que vêm sendo utilizadas para substituir a gordura em hambúrgueres. Inglett (1996) verificou, em estudo, que um preparado contendo casca de aveia, casca de arroz, vagem de soja, ervilhas e farelo de trigo ou milho, conhecido comercialmente como Z-TRIM, forneceu ao hambúrguer uma textura macia.

Ao estudarem a influência da adição de fécula de mandioca e farinha de aveia em hambúrguer de carne ovina, Seabra et al. (2002) obtiveram resultados revelando que as formulações com $2 \%$ de fécula de mandioca, assim como aquela adicionada de $2 \%$ de farinha de aveia, apresentaram menor teor de gordura, antes e depois de cozidos, menor encolhimento, maior rendimento e capacidade de retenção de água, ao mesmo tempo em que apresentaram problemas sensoriais, com relação a suculência, maciez e textura. Os resultados dessa pesquisa já indicavam o grande desafio tecnológico da adição de fibras em produtos cárneos, uma vez que, nesse caso, há tendência de o produto apresentar textura firme, o que exige maior esforço durante a mastigação.

Costa (2004) comentou que a utilização de uma mistura de farelo e fibra de aveia com condimentos, em hambúrguer com 10,0 \% de gordura, apresentou rendimento superior (15,0\%) no cozimento ao do controle, com $20,0 \%$ de gordura. O estudo obteve resultados que demonstraram que o farelo e a fibra de aveia apresentam boa capacidade de retenção de água e podem imitar a cor e a textura da gordura. A autora relatou ainda que produtos à base de proteína de soja (farinha de soja, concentrado proteico de soja e proteína de soja) são também comumente utilizados como extensores ou ligantes em produtos cárneos, tais como hambúrgueres.

A importância da redução de gorduras foi exaltada por Hadorn et al. (2008), ao estudarem a variação dos percentuais de gorduras em salsichas, salame, bacon e hambúrguer, pois constataram resultados insignificantes nas propriedades tecnológicas desses produtos. Os resultados sensoriais obtidos por Dias et al. (2009) para um produto 'tipo' hambúrguer, substituindo a carne bovina por soja, enriquecido com linhaça e quinoa, não foram satisfatórios. Uma possível justificativa para a baixa aceitação desse hambúrguer desenvolvido pode estar relacionada ao fato de os provadores esperarem sabor de carne no produto, que geralmente é associado a esse tipo de alimento.

Em estudo realizado por García et al. (2009), foi constatado que a adição de casca de tomate seco em hambúrgueres pode ser útil para obtenção de um novo produto enriquecido com licopeno (4,9 mg/100g), além de proporcionar uma utilização para este subproduto da indústria do tomate.

Santos Júnior et al. (2009) também obtiveram resultados favoráveis com relação à redução dos teores de gordura em formulações de hambúrguer de carne ovina por meio da suplementação de farinha de aveia, tendo sido observada maior aceitação sensorial na formulação adicionada de 4 \% da fibra. Embora Bourscheid (2009) não tenha verificado redução significativa do teor de gordura em hambúrgueres adicionados de fécula de mandioca e proteína texturizada de soja, ele constatou menor percentual de encolhimento e maior percentual de rendimento para essas formulações após cocção, fatores economicamente esperados.

Diferentemente dos resultados obtidos por Dias et al. (2009), a avaliação sensorial de hambúrgueres de proteína texturizada de soja e atum revelou grande aceitação do produto, reforçando o potencial de alimentos à base de soja, quando combinados com outras matériasprimas (ALBUQUERQUE et al., 2009). Nesse caso, a boa aceitação do produto pode ser em função do sabor diferenciado proveniente da adição de atum, o qual pode ter contribuído para mascarar o sabor e o aroma da soja.

Galán et al. (2010) testaram como alternativa para um hambúrguer mais saudável a adição de ácido fólico em diferentes concentrações (0,6, 1,2 e 2,4 mg/100g). Os autores obtiveram resultados que permitiram concluir que a adição de ácido fólico nas diferentes concentrações não interferiu sobre a cor e a textura, atributos estes importantes para a qualidade sensorial do produto. Resultados também satisfatórios foram verificados por 
Almeida (2011), que concluiu que a adição de diferentes níveis de farinha de aveia em hambúrguer de carne caprina não interferiu na capacidade de retenção de água dos mesmos, sendo comprovado estatisticamente que, com uma concentração de 4,0 \% de farinha de aveia, ocorreu menor perda de peso por cozimento $(18,4 \%)$ em relação ao grupo controle; o mesmo ocorreu com os teores de lipídios, uma vez que ficou comprovado que a adição de farinha de aveia contribuiu para a redução de gordura dos hambúrgueres adicionados dessa fonte de fibras. Youssef e Barbut (2011) obtiveram bons resultados de textura e rendimento em hambúrgueres com a substituição de gordura animal por óleo vegetal (canola).

Bernardino Filho et al. (2012) verificaram um percentual de gorduras totais na ordem de $2,41 \%$ em hambúrgueres contendo $50 \%$ de inulina (fibra dietética solúvel extraída comercialmente da raiz da chicória) e 50 $\%$ de toucinho; esse resultado mostrou-se semelhante aos encontrados por Marques (2007), que estudou o efeito da adição de 12,25 \% de farinha de aveia como substituto de gordura animal em hambúrguer de carne bovina, encontrando $2,45 \%$ de gordura nos produtos crus.

Em estudo recente (MARTINEZ et al., 2012), foi observado que a adição de uma mistura de óleos de oliva, milho e de peixe em carne para hambúrguer implicou em resultados que se caracterizam como alternativa viável para a redução do teor de lipídios totais e a incorporação de ácidos graxos poli-insaturados (ômegas-3 e 6), além de ter sido evidenciada boa aceitação das formulações desenvolvidas durante a avaliação sensorial das mesmas.

Segundo Muhlisin et al. (2013), além de propriedades antioxidantes, o alecrim pode atuar no organismo como agente anti-inflamatório e ajudar na prevenção e no tratamento de problemas do sistema digestivo, bem como melhorar os sintomas de constipações intestinais. Por isso, os pesquisadores desenvolveram formulações de hambúrgueres adicionados de extrato de alecrim, lactato de cálcio e lactato de sódio, aditivos estes que contribuíram para os resultados satisfatórios, aumentando a vida de prateleira do produto.

Embora a literatura acumule trabalhos sobre a substituição parcial ou total de gordura saturada em diferentes produtos cárneos, inclusive em hambúrguer, não foi localizada publicação científica, na qual tivesse sido estudada a influência da redução de cloreto de sódio por meio da sua substituição, parcial ou total, por outro tipo de sal em hambúrguer.

Apesar de não terem estudado a influência da redução do conteúdo de sódio nas características dos hambúrgueres desenvolvidos, López-López et al. (2010) demonstraram preocupação em tornar o produto mais saudável, acrescentando nas formulações somente 0,5 $\%$ de cloreto de sódio, quando o comum é acrescentar $2,0 \%$.

\subsubsection{Patentes de hambúrgueres com características funcionais}

Com a intenção de oferecer ao mercado hambúrgueres com características funcionais, algumas patentes foram depositadas. A exemplo disso, citam-se algumas formulações desses produtos, como, por exemplos: massa cárnea com adição de fécula de mandioca e proteínas lácteas, e vegetais para elaboração de vários produtos cárneos, entre os quais, o hambúrguer (SOUZA, 2004); massa cárnea para hambúrguer adicionada de chá verde e óleo de gergelim (JEONG, 2008); formulação de hambúrguer bovino adicionado de ácido graxo ômega-3 (GOMES, 2010); formulação de carne vegetal para hambúrguer contendo gordura vegetal (livre de ácidos graxos 'trans') e Okara (resíduo da produção de leite de soja) (MORETTI, 2010); hambúrguer de palmito com adição de isolado proteico de soja e gordura vegetal (FANTINI, 2011), e hambúrguer de vegetais recheado, congelado e adicionado de farinha de arroz e proteína de soja (XIAOGONG et al., 2011).

\subsubsection{Farinha de linhaça dourada: fonte de fibras e ácidos graxos insaturados}

As gorduras insaturadas apresentam-se nas formas monoinsaturada (apenas uma dupla ligação na molécula) ou poli-insaturada (mais de uma dupla ligação na molécula). Segundo Youdin et al. (2000) apud Martin et al. (2006), os ácidos graxos poli-insaturados abrangem as famílias de ácidos graxos ômega-3 e ômega-6, estando presentes principalmente em cereais e leguminosas, tais como aveia, ervilha, soja, amêndoa, castanha-dopará, canola e semente de linhaça; estes possuem funções essenciais para manter, sob condições normais, as membranas celulares, a transmissão de impulsos nervosos, as funções cerebrais, a transferência do oxigênio atmosférico para o plasma sanguíneo, a divisão celular e a síntese de hemoglobina. São denominados essenciais, pois não podem ser sintetizados pelo organismo humano.

A semente de linhaça, que contém de 30 a $40 \%$ de gordura, de 20 a $25 \%$ de proteína e de 20 a $28 \%$ de fibra em sua composição, tem sido atualmente muito consumida em virtude das suas propriedades benéficas, sendo considerada, dentre os vegetais, a principal fonte de ácidos graxos ômega-3, constituindo cerca de 50 $\%$ do total de gordura encontrado na semente (ROLIM, 2007). O autor comentou ainda que, além de suas funções nutricionais básicas, a semente de linhaça produz efeitos metabólicos e fisiológicos benéficos à saúde, porque apresenta rico conteúdo de ácidos graxos $\alpha$-linolênico e linoleico, precursores de outros ácidos graxos das séries ômega-3 (n-3) e ômega-6 (n-6), respectivamente, o que permite considerá-la um alimento funcional. 
Os AG n-6 e n-3 devem ser muito bem diferenciados, pois são metabolicamente diferentes e possuem funções fisiológicas opostas. Desse modo, o equilíbrio nutricional é importante para se conseguir a homeostasia e o desenvolvimento normal do organismo. Um balanço adequado na proporção de n-6:n-3 na dieta é essencial no metabolismo do organismo humano, podendo levar à prevenção de doenças cardiovasculares e crônicas degenerativas, e também a uma melhor saúde mental (NOVELLO et al., 2008).

Além disso, a semente de linhaça também é rica em compostos fenólicos, que agem como antioxidantes, e ligninas, substâncias com estrutura química muito semelhante ao estrogênio, exercendo atividade semelhante à desse hormônio. Em função de tal característica, a semente de linhaça costuma ser muito utilizada para minimizar os sintomas da menopausa, período em que os níveis de estrogênio são naturalmente diminuídos. A proteína presente na linhaça é semelhante à da soja, caracterizando-a como uma proteína completa. Já as fibras alimentares encontradas apresentam uma boa proporção entre a fibra solúvel e a insolúvel, auxiliando, portanto, na diminuição do colesterol e no bom funcionamento do intestino (ROLIM, 2007).

Nesse contexto, a adição de farinha de linhaça dourada como substituto de gordura animal e fonte de fibras e ácidos graxos insaturados em hambúrguer de carne bovina, bem como a redução do seu conteúdo de sódio, podem caracterizar alternativas para um produto cárneo mais saudável.

\section{Conclusões}

A mais nova geração de substitutos de gordura em produtos cárneos tem incentivado pesquisas que visam ao desenvolvimento de hambúrgueres com baixo conteúdo calórico, em razão, principalmente, da demanda proveniente de consumidores preocupados com a saúde. Essa demanda tem permitido o avanço tecnológico, porém, melhorias com relação à funcionalidade e à palatabilidade desses produtos cárneos adicionados de fibras devem ser mais investigadas pelas comunidades industrial e científica.

A mesma preocupação deve ser dada com relação ao consumo excessivo de sal. Até o momento, somente uma pesquisa propôs a redução de sódio em hambúrgueres; porém, não a sua substituição parcial por outro sal, já que sais contribuem para melhor estabilidade microbiológica do alimento. Esses motivos indicam a necessidade de novos estudos que verifiquem a influência da redução desse constituinte através de diferentes proporções de substituição por outros tipos de sais e a sua comparação com o produto convencional.

\section{Referências}

AKOH, C. C. Fat replacers. Food Technology, Chicago, v. 52, n. 3, p. 47-53, 1998.

ALBUQUERQUE, T. L.; LIMA, M. D.; OLIVEIRA, V. S.; COELHO, R. M. D.; RODRIGUES, M. D. P. Processing and sensorial acceptance of hamburguer type product consisted of soy (Glycine max) and tuna (Thunnus spp). Boletim do CEPPA, Curitiba, v. 27, n. 2, p. 191-198, 2009.

ALMEIDA, R. S. Processamento de Hambúrguer de Carne Caprina Adicionados com Diferentes Níveis de Farinha de Aveia. 2011. 73 f. Dissertação (Mestrado em Zootecnia)Universidade Estadual do Sudoeste da Bahia, Itapetinga, 2011.

AMERICAN DIETETIC ASSOCIATION - ADA. Position of the American Dietetic Association: fat replacers. Journal of American Dietetic Association, Houston, v. 67, n. 105, p. 266-275, 2005

ANDERSON, E. T.; BERRY, B. W. Effects of inner pea fiber on fat retention and cooking yield in hight ground beef. Food Research International, Beltsville, v. 1, n. 1, p. 1-7, 2001. http://dx.doi. org/10.1016/S0963-9969(01)00089-8

ARAÚJO, E. M.; MENEZES, H. C.; TOMAZINI, J. M. Fibras solúveis e insolúveis de verduras, tubérculos e canela para uso em nutrição clínica. Ciência e Tecnologia de Alimentos, Campinas, v. 29, n. 2, p. 401-406, 2009. http://dx.doi. org/10.1590/S0101-20612009000200027

BARBOSA, L. N. Propriedade Antimicrobiana de Óleos Essenciais de Plantas Condimentares com Potencial de uso como Conservante em Carne e Hambúrguer Bovino e Testes de Aceitação. 2010. 121 f. Dissertação (Mestrado em Biologia Geral e Aplicada)-Instituto de Biociências, Universidade Estadual Paulista Júlio de Mesquita Filho, Botucatu, 2010.

BARBOSA, M. Na onda da barra: Com uma gestão alternativa e um produto campeão de vendas, Nutrimental afasta a crise e volta a ser uma empresa saudável. Isto é Dinheiro, São Paulo, n. 224, 30 nov. 2001. Disponível em: <http://www.terra.com.br/ istoedinheiro>. Acesso em: 04 maio 2013.

BAXTER, Y. C. Fibras alimentares: nutriente funcional na prevenção e tratamento da obstipação intestinal. Boletim da Sociedade Brasileira de Nutrição Parenteral e Enteral, Rio de Janeiro, v. 32, n. 1, p. 18-19, 2001

BERNARDINO FILHO, R.; OLIVEIRA, S. P.; GOMES, Q. O. Elaboração de hambúrguer bovino adicionado de inulina como ingrediente funcional prebiótico e substituto de gordura. Revista Verde de Agroecologia e Desenvolvimento Sustentável, Mossoró, v. 7, n. 4, p. 33-37, 2012.

BOCK, M.; DERRAIK, J. G. B.; BRENNAN, C. M.; BIGGS, J. B.; SMITH, G. C.; CAMERON-SMITH, D.; WALL, C. R.; CUTFIELD, W S. Psyllium Supplementation in Adolescents Improves Fat Distribution \& Lipid Profile: A Randomized, Participant-Blinded, 
Alternativas para um produto cárneo mais saudável: uma revisão

OLIVEIRA, D. F. et al.

Placebo-Controlled, Crossover Trial. PLoS ONE, San Francisco, v. 7, n. 7, p. 417-435, 2012.

BOURSCHEID, C. Avaliação da Influência da Fécula de Mandioca e Proteína Texturizada de Soja nas Características Físico - Químicas e Sensoriais de Hambúrguer de Carne Bovina. 2009. 52 f. Trabalho de Conclusão de Curso (Bacharelado em Engenharia de Alimentos) - Universidade do Estado de Santa Catarina, Pinhalzinho, 2009.

BOWLES, S.; DEMIATE, I. M. Caracterização físico-química de okara e aplicação em pães do tipo francês. Ciência e Tecnologia de Alimentos, Campinas, v. 26, n. 3, p. 652-659, 2006. http://dx.doi.org/10.1590/S0101-20612006000300026

BRAGAGNOLO, N.; RODRIGUEZ-AMAYA, D. B. Teores de colesterol, lipídios totais e ácidos graxos em cortes de carne suína. Ciência e Tecnologia de Alimentos, Campinas, v. 22, n. 1, p. 1037-1043, 2002. http://dx.doi.org/10.1590/S010120612002000100018

BRASIL. Ministério da Saúde. Portaria n 398, de 30 de abril de 1999. Regulamento Técnico que Estabelece as Diretrizes Básicas para Análise e Comprovação de Propriedades Funcionais e ou de Saúde Alegadas em Rotulagem de Alimentos. Diário Oficial da República Federativa do Brasil, Brasília, 3 maio 1999. Seção 1. Disponível em: <http://e-legis.bvs.br/ leisref/public/showAct.php?id=11297\&mode=PRINTVERSION $>$. Acesso em: 29 abr. 2013.

BRASIL. Ministério da Agricultura, Pecuária e Abastecimento. Instrução Normativa $n^{\circ} 20$, de 31 de julho de 2000. Regulamentos Técnicos de Identidade e Qualidade de Almôndega, de Apresuntado, de Fiambre, de Hamburguer, de Kibe, de Presunto Cozido e de Presunto. Diário Oficial da República Federativa do Brasil, Brasília, DF, 1 ago. 2000. Seção 1, p. 3-31.

BRASIL. Ministério da Saúde. Guia Alimentar para a População Brasileira - Promovendo a Alimentação Saudável. Brasília: Ministério da Saúde, 2006. Disponível em: <http://nutricao. saude.gov.br/guia_conheca.php>. Acesso em: 29 abr. 2013.

BUSCH, J. L. H. C.; YONG, F. Y. S.; GOH, S. M. Sodium reduction: Optimizing product composition and structure towards increasing saltiness perception. Trends in Food Science \& Technology, Oxford, v. 29, n. 1, p. 21-34, 2013. http://dx.doi. org/10.1016/j.tifs.2012.08.005

CATALANI, L. A. Fibras alimentares. Revista Brasileira de Nutrição Clínica, São Paulo, v. 18, n. 4, p. 178-182, 2003

CERCATO, C.; MANCINI, M. C.; ARGUELLO, A. M. C.; PASSOS, V. Q.; VILLARES, S. M. F.; HALPERN, A. Systemic hypertension, diabetes mellitus, and dyslipidemia in relation to body mass index: evaluation of a Brazilian population. Revista do Hospital das Clínicas Faculdade de Medicina - USP, São Paulo, v. 59, n. 3, p. 113-118, 2004. http://dx.doi.org/10.1590/S004187812004000300004
CIRIANO, M. G. I.; BERASATEGI, I.; NAVARRO-BLASCO, I.; ASTIASARAN, I.; ANSORENA, D. Reduction of sodium and increment of calcium and omega-3 polyunsaturated fatty acids in dry fermented sausages: effects on the mineral content, lipid profile and sensory quality. Journal of the Science of Food and Agriculture, London, v. 93, n. 4, p. 876-881, 2013. http://dx.doi. org/10.1002/jsfa.5811

COLMENERO, J. F. Relevants factors in strategies for fat reduction meat products. Food Science \& Technology, Amsterdam, v. 11, n. 2, p. 56-66, 2000. http://dx.doi.org/10.1016/ S0924-2244(00)00042-X

COORDENAÇÃO DE APERFEIÇOAMENTO DE PESSOAL DE NÍVEL SUPERIOR - CAPES. Portal de Periódicos Capes. Disponível em: <http://www.periodicos.capes.gov.br/portugues/ index.jsp>. Acesso em: 28 abr. 2013.

COSTA, L.O. Processamento e Diminuição do Reprocesso do Hambúrguer Bovino (HBV). 2004. 127 f. Trabalho de Conclusão de Curso (Graduação em Engenharia de Alimentos)Universidade Católica de Goiás, Goiânia, 2004.

COXSON, P. G.; COOK, N. R.; JOFFRES, M.; HONG, Y. L.; ORENSTEIN, D.; SCHMIDT, S. M.; BIBBINS-DOMINGO, K. Mortality Benefits From US Population-wide Reduction in Sodium Consumption Projections From 3 Modeling Approaches. Hypertension, Lancet, v. 61, n. 3, p. 564-570, 2013. http://dx.doi. org/10.1161/HYPERTENSIONAHA.111.201293

DIAS, V. M.; DIAS, K. M.; PILLA, V. Desenvolvimento e análise sensorial de hambúrger de soja enriquecido com linhaça e quinoa. In: ENCONTRO LATINO AMERICANO DE INICIAÇÃO CIENTÍFICA E ENCONTRO LATINO AMERICANO DE PÓS-GRADUAÇÃO, 9.13., 2009, Vale do Paraíba. Anais eletrônicos... Vale do Paraíba: Universidade do Vale do Paraíba, 2009. Disponível em: <http://www.inicepg.univap.br/ cd/INIC_2009/anais/arquivos/RE_0409_1259_01.pdf >. Acesso em: 10 maio 2013.

FANTINI, E. L. Hambúrguer de Palmito. BR n. PI 1000785-7 A2, 2011. Disponível em: <http://www. patentesonline.es/>. Acesso em: 13. Maio 2013.

FERREIRA, L. G.; BURINI, R. C.; MAIA, A. F. Dietas vegetarianas e desempenho esportivo. Revista de Nutrição, Campinas, v. 19, n. 4, p. 469-477, 2006. http://dx.doi.org/10.1590/S141552732006000400006

FRANCISCHI, R. P. P.; PEREIRA, L. O.; FREITAS, C. S.; KLOPFER, M.; SANTOS, R. C.; VIEIRA, R. C.; VIEIRA, P.; LANCHA JÚNIOR, A. H. Obesidade: atualização sobre sua etiologia, morbidade e tratamento. Revista de Nutrição, Campinas, v. 13, n. 1, p. 17-28, jan/abr 2000. http://dx.doi.org/10.1590/S141552732000000100003

FREITAS, D. G. C.; MORETTI, R. H. Caracterização e avaliação sensorial de barra de cereais funcional de alto teor protéico e vitamínico. Ciência e Tecnologia de Alimentos, Campinas, 
Alternativas para um produto cárneo mais saudável: uma revisão

OLIVEIRA, D. F. et al.

v. 26, n. 2, p. 318-324, 2006. http://dx.doi.org/10.1590/S010120612006000200014

FREITAS, O. C.; CARVALHO, F. R.; NEVES, J. M.; VELUDO, P. K.; PARREIRA, R. S.; GONÇALVES, R. M.; LIMA, S. A.; BESTETTI, R. B. Prevalence of Hypertension in the urban population of Catanduva, in the state of São Paulo, Brazil. Arquivo Brasileiro de Cardiologia, São Paulo, v. 77, n. 2, p. 16-21, 2001. http:// dx.doi.org/10.1590/S0066-782X2001000700002

GALÁN, I.; GARCÍA, M. L.; SELGAS, M. D. Effects of irradiation on hamburgers enriched with folic acid. Meat Science, Barking, v. 84, n. 3, p. 437-443, 2010. http://dx.doi.org/10.1016/j. meatsci.2009.09.013

GARCÍA, M. L.; CALVO, M. M.; SELGAS, M. D. Beef hamburgers enriched in lycopene using dry tomato peel as an ingredient. Meat Science, Barking, v. 83, n. 3, p. 45-49, 2009. http://dx.doi. org/10.1016/j.meatsci.2009.03.009

GIUNTINI, E. B.; LAJOLO, F. M.; MENEZES, E. W. Composição de alimentos: um pouco de história. Archivos Latinoamericanos de Nutrición, Caracas, v. 56, n. 3, p. 45-54, 2006.

GOMES, E. V. Formulação de Hambúrguer Bovino, Embutido Cozido de Carne Bovina, Presunto e Salsicha, Adicionados de Ácido Graxo do Tipo Ômega-3, e Respectivos Processos de Obtenção. BR n. PI 0802542-8 A2, 2010. Disponível em: <http://www.patentesonline.es/>. Acesso em: 13. Mai. 2013.

GOOGLE. Google Acadêmico. Disponível em: <http://scholar. google.com.br/schhp?=pt-BR>. Acesso em: 28 abr. 2013.

GUTKOSKI, L. C.; BONAMIGO, J. M. A.; TEIXEIRA, D. M. A.; PEDÓ, I. Desenvolvimento de barras de cereais à base de aveia com alto teor de fibra alimentar. Ciência e Tecnologia de Alimentos, Campinas, v. 27, n. 2, p. 355-363, 2007. http:// dx.doi.org/10.1590/S0101-20612007000200025

HADORN, R.; EBERHARD, P.; GUGGISBERG, D.; PICCINALI, P.; SCHLICHTHERLE-CERNY, H. Effect of fat score on the quality of various meat products. Meat Science, Barking, v. 80, n. 3, p. 765-770, 2008. http://dx.doi.org/10.1016/j. meatsci.2008.03.020

HASLER, C. M. Functional foods: their role in disease in: developing new food products for a changing prevention and health promotion. Food Technology, Chicago, v. 52, n. 2. p.5762, 1998.

HAUTRIVE, T. P.; OLIVEIRA, V. R.; SILVA, A. R. D.; TERRA, N. N.; CAMPAGNOL, P. C. B. Análise físico-química e sensorial de hambúrguer elaborado com carne de avestruz. Ciência e Tecnologia de Alimentos, Campinas, v. 28, n. 2, p. 95-101, 2008. http://dx.doi.org/10.1590/S0101-20612008000500016

INGLETT, G. E. New Trim from Trash and More. Washington: Food \& Nutrition Research Briefs, Index: Agricultural Research Service (ARS), 1996. Disponível em: <http://www.ars.usda.gov/ is/np/fnrb/fnrb1096.html>. Acesso em: 13 maio 2013.
JENSEN, M.; SMITH, G.; FEAR, S.; SCHILMOELLER, L.; JOHNSON, C. Seasoning and Method for Seasoning a Food Product While Reducing Dietary Sodium Intake. US n. 2008/0003344, 2008. Disponível em: <http://www. freepatentsonline.com/y2008/0003344.html>. Acesso em: 28 abr. 2013.

JEONG, L. W. Burger Patty Chocos. US n. KR100851037 B1, 2008. Disponível em: <http://lp.espacenet.com/>. Acesso em: 13 maio 2013.

KARANJA, N.; LANCASTER, K. J.; VOLLMER, W. M.; LIN, P. H.; MOST, M. M.; ARD, J. D.; SWAIN, J. F.; SACKS, F. M.; OBARZANEK, E. Acceptability of sodium-reduced research diets, including the dietary approaches to stop hypertension diet, among adults with prehypertension and stage 1 hypertension. Journal American Dietetic Associaction, Philadelphia, v. 107, n. 9, p. 1530-1538, 2007. http://dx.doi.org/10.1016/j. jada.2007.06.013

KWAK, N.; JUKES, D. J. Functional foods. Part 1: the development of a regulatory concept. Food Control, v. 12, n. 3, p. 99-107, 2001. http://dx.doi.org/10.1016/S0956-7135(00)00028-1

LATIPAT-ESPACENET. Patentes Latinoamericanas. Disponível em: <http://lp.espacenet.com/>. Acesso em: 13 maio 2013.

LI, N.; PRESCOTT, J.; WU, Y.; BARZI, F.; YU, X.; ZHAO, L.; NEAL, B. The effects of a reduced-sodium, high-potassium salt substitute on food taste and acceptability in rural northern China. British Journal of Nutrition, Cambridge, v. 101, n. 3, p. 10881093, 2009. http://dx.doi.org/10.1017/S0007114508042360

LÓPEZ-LÓPEZ, I.; COFRADES, S.; RUIZ-CAPILLAS, C.; JIMÉNEZ-COLMENERO, F. Design and nutritional properties of potential functional frankfurters based on lipid formulation, added seaweed and low salt content. Meat Science, Barking, v. 83, n. 2, p. 255-262, 2010. http://dx.doi.org/10.1016/j. meatsci.2009.05.014

MARIATH, A. B.; GRILLO, L. P.; SILVA, R. O.; SCHMITZ, P.; CAMPOS, I. C.; MEDINA, J. R. P.; KRUGER, R. M. Obesidade e fatores de risco para o desenvolvimento de doenças crônicas não transmissíveis entre usuários de unidade de alimentação e nutrição. Caderno de Saúde Pública, Rio de Janeiro, v. 23, n. 4, p. 897-905, 2007. http://dx.doi.org/10.1590/S0102$311 \times 2007000400017$

MARQUES, J. M. Elaboração de um Produto de Carne Bovina "Tipo Hambúrguer" Adicionado de Farinha de Aveia. 2007. 71 f. Dissertação (Mestrado em Tecnologia de Alimentos)Universidade Federal do Paraná, Curitiba, 2007.

MARTIN, A. C.; ALMEIDA, V. V.; RUIZ, R. M.; VISENTAINER, L. E. J.; MATSHUSHITA, M.; SOUZA, E. N.; VISENTAINER, V. J. Ácidos graxos poliinsaturados ômega-3 e ômega-6: importância e ocorrência em alimentos. Revista de Nutrição, Campinas, v. 19, n. 6, p. 661-670, 2006. http://dx.doi.org/10.1590/S141552732006000600011 
Alternativas para um produto cárneo mais saudável: uma revisão

OLIVEIRA, D. F. et al.

MARTINEZ, B.; MIRANDA, J. M.; VAZQUEZ, B. I.; FENTE, C. A.; FRANCO, C. M.; RODRIGUEZ, J. L.; CEPEDA, A. Development of a Hamburger Patty with Healthier Lipid Formulation and Study of its Nutritional, Sensory, and Stability Properties. Food and Bioprocess Technology, New York, v. 5, n. 1, p. 200-208, 2012. http://dx.doi.org/10.1007/s11947-009-0268-x

MATTES, R. D. Position of the American Dietetic Association: fat replacers. Journal American Dietetic Associaction, Philadelphia, v. 98, n. 4, p. 463-468, 1998. http://dx.doi. org/10.1016/S0002-8223(98)00105-9

MONEGO, M. A. Goma da Linhaça (Linun usitatissimum L.) para Uso como Hidrocolóide na Indústria de Alimentos. 2009. 89 f. Dissertação (Mestrado em Ciência e Tecnologia de Alimentos) - Centro de Ciências Rurais, Universidade Federal de Santa Maria, Santa Maria, 2009.

MORAES, F. P.; COLLA, L. M. Alimentos funcionais e nutracêuticos: definições, legislação e benefícios à saúde. Revista Eletrônica de Farmácia, Goiânia, v. 3, n. 2, p. 109-122, 2006. Disponível em <http://revistas.ufg.br/index.php/REF/ article/viewFile/2082/2024>. Acesso em: 04 maio 2013.

MORETTI, R. H. Formulação de Carne Vegetal. BR n. PI 0805504-1 A2, 2010. Disponível em: <http://www. patentesonline. es/>. Acesso em: 13 maio 2013.

MUHLISIN, M.; KANG, S. M.; CHOI, W. H.; LEE, K. T.; CHEONG, S. H.; LEE, S. K. The effect of modified atmosphere packaging and addition of rosemary extract, sodium acetate and calcium lactate mixture on the quality of pre-cooked hamburger patties during refrigerated storage. Asian-Australasian Journal of Animal Sciences, Korea, v. 26, n. 1, p. 134-142, 2013. http:// dx.doi.org/10.5713/ajas.2012.12468

NASCIMENTO, R.; CAMPAGNOL, P. C. B.; MONTEIRO, E. S.; POLLONIO, M. A. R. Substituição de cloreto de sódio por cloreto de potássio: influência sobre as características físico-químicas e sensoriais de salsichas. Alimentos e Nutrição, Araraquara, v. 18, n. 3, p. 297-302, 2007.

NOONAN, W. P.; NOONAN, C. Legal requeriments for "functional foods" claims. Toxicology Letters, Netherlands, v. 150, n. 4, p. 19-24, 2004. http://dx.doi.org/10.1016/j.toxlet.2003.05.002

NOVELLO, D.; FRANCESCHINI, P.; QUINTILIANO, D. A. A importância dos ácidos graxos $\omega$-3 e $\omega$-6 para a prevenção de doenças e na saúde humana. Salus, Guarapuava, v. 2, n. 1 , p. $80,2008$.

ORTIGOZA, S. A. G. Alimentação e saúde: as novas relações espaço-tempo e suas implicações nos hábitos de consumo de alimentos. RA'E GA - O Espaço Geográfico em Análise, Curitiba, n. 15, p. 83-93, 2008. Disponível em: <http://ojs.c3sl. ufpr.br/ojs2/index.php/raega/article/view/14247/9573. Acesso em: 14 maio 2013.

PATENTESONLINE.ES. Patentes Internacionais. Disponível em: <http://www.patentesonline.es/. Acesso em: 13 maio 2013.
PAULINO, F. O.; SILVA, T. J. P.; FRANCO, R. M.; FREITAS, M. Q.; FERNANDES, M. L. Redução parcial dos teores de gordura e sal em embutido cárneo suíno com utilização de goma carragena e cloreto de potássio. Revista Brasileira de Ciência Veterinária, Niterói, v. 13, n. 2, p. 121-124, 2006.

PHILLIPS, F. Don't pass the salt. Food and Nutrition Bulletin, Tóquio, v. 28, n. 3, p. 339-40, 2003.

PIÑERO, M. P.; PARRA, K.; HUERTA-LEIDENS, N.; MORENO, L. A.; FERRER, M.; ARAUJO, S.; BARBOZA, Y. Effect of oat's soluble fibre ( $\beta$-glucan) as a fat replacer on physical, chemical, microbiological and sensory properties on low-fat beef patties. Meat Science, Barking, v. 80, n. 3, p. 678-680, 2008. http:// dx.doi.org/10.1016/j.meatsci.2008.03.006

PINHEIRO, M. V. S.; PENNA, A. L. B. Substitutos de gordura: tipos e aplicações em produtos lácteos. Alimentos e Nutrição, Araraquara, v. 15, n. 2, p. 175-186, 2004.

PROENÇA, R. P. C. Alimentação e globalização: algumas reflexões. Ciência e Cultura, Campinas, v. 62, n. 4, p. 43-47, 2010.

QI, L.; MEIGS, J. B.; LIU, S.; MANSON, J. E.; MANTZOROS, C.; $\mathrm{HU}, \mathrm{F}$. B. Dietary fibers and glycemic load, obesity, and plasma adiponectin levels in women with type 2 diabetes. Diabetes Care, Alexandria, v. 29, n. 7, p. 1501-1505, 2006. http://dx.doi. org/10.2337/dc06-0221

QUEIROZ, Y. U.; DAUD, K. O.; SOARES, R. A. M.; SAMPAIO, G. R.; CAPRILES, V. D.; TORRES, E. A. F. S. Desenvolvimento e avaliação das propriedades físico-químicas de hambúrgueres com reduzidos teores de gordura e de colesterol. Revista Nacional da Carne, São Paulo, v. 338, n. 2, p. 84-89, 2005.

REZENDE, R.; ROSADO, L.; FRANCESCHINNI, S.; ROSADO, G.; RIBEIRO, R.; MARINS, J. C. B. Revisão crítica dos métodos disponiveis para avaliar a composição corporal em grandes estudos populacionais e clínicos. Archivos Latinoamericanos de Nutrición, Caracas, v. 57, n. 4, p. 327-334, 2007

RODRÍGUEZ, R.; JIMÉREZ, A.; FERÑANDEZ-BOLAÑOS, J.; GUILLÉN, R.; HEREDIA, A. Dietary fibre from vegetable products as source of functional ingredients. Trends in Food Science \& Technology, Oxford, v. 17, n. 1, p. 3-15, 2006. http://dx.doi. org/10.1016/j.tifs.2005.10.002

ROLIM, S. S. Nutrição e Saúde. 2007. Disponível em: <www. nutrociencia.com.br> Acesso em: 13 maio 2013.

SALES, R. L.; VOLP, A. C. P.; BARBOSA, K. B. F.; DANTAS, M. I. S.; DUARTE, H. S.; MINIM, V. P. R. Mapa de preferência de sorvetes ricos em fibras. Ciência e Tecnologia de Alimentos, Campinas, v. 28, n. 1, p. 27-31, 2008. http://dx.doi.org/10.1590/ S0101-20612008000500005

SANTOS JÚNIOR, L. C. O.; RIZZATTI, R.; BRUNGERA, A.; SCHIAVINI, T. J.; CAMPOS, E. F. M.; SCALCO NETO, J. F.; RODRIGUES, L. B.; DICKEL, E. L.; SANTOS, L. R. Desenvolvimento de hambúrguer de carne de ovinos de 
Alternativas para um produto cárneo mais saudável: uma revisão

OLIVEIRA, D. F. et al.

descarte enriquecido com farinha de aveia. Ciência Animal Brasileira, Goiânia, v. 10, n. 4, p. 1128-1134, 2009.

SARTORELLI, D. S.; FRANCO, L. J. Tendências do diabetes mellitus no Brasil: o papel da transição nutricional. Cadernos de Saúde Pública, Rio de Janeiro, v. 19, n. 1, p. 29-36, 2003. http://dx.doi.org/10.1590/S0102-311X2003000700004

SCIENCE DIRECT. Busca bibliográfica. Disponível em: <http:// www.sciencedirect.com>. Acesso em: 28 abr. 2013.

SCIENTIFC ELETRONIC BASE LIBRARY ONLINE - ScIELO. Disponível em: <http://www.scielo.org/php/index.php>. Acesso em: 28 abr. 2013.

SEABRA, L. M. J.; ZAPATA, J. F. F.; NOGUEIRA, C. M.; DANTAS, M. A.; ALMEIDA, R. B. Fécula de mandioca e farinha de aveia como substitutos de gordura na formulação de hambúrguer de carne ovina. Ciência e Tecnologia de Alimentos, Campinas, v. 22, n. 3, p. 244-248, 2002. http://dx.doi.org/10.1590/S010120612002000300008

SICHIERI, R.; COITINHO, D. C.; MONTEIRO, J. B.; COUTINHO, W. F. Recomendações de Alimentação e Nutrição Saudável para a População Brasileira. Arquivo Brasileiro de Endocinologia e Metabologia, São Paulo, v. 44, n. 3, p. 227-232, 2000. http:// dx.doi.org/10.1590/S0004-27302000000300007

SOUZA, A.; MEDEIROS, A. R.; SOUZA, A. C.; WINK, M.; SIQUEIRA, I. R.; FERREIRA, M. B. C.; FERNANDES, L.; LOAYZA HIDALGO, M. P.; TORRES, I. L. S. Avaliação do impacto da exposição a agrotóxicos sobre a saúde de população rural do
Vale do Taquari (RS, Brasil). Ciência e Saúde Coletiva, Rio de Janeiro, v. 16, n. 8, p. 122-130, 2011. http://dx.doi.org/10.1590/ S1413-81232011000900020

SOUZA, D. F. Produtos para Segmento Frigorífico e Processo de Obtenção desses Produtos. BR n. PI 0303197-7 A2, 2004. Disponível em: <http://www.patentesonline.es/>. Acesso em: 13 maio 2013.

TORRES, G. F.; SALGADO, S. M.; LIVERA, A. V. S.; GUERRA, N. B. Efeito do processo hidrotérmico sobre o teor de fibra alimentar em hortaliças. Boletim do CEPPA, Curitiba, v. 24, n. 2, p. 237-346, 2006.

WEB OF KNOWLEDGE. Disponível em: <http://www. webofknowledge.com>. Acesso em: 28 abr. 2013.

XIAOGONG, Z.; JINFENG, L.; YAJING, W. Vegetable Burger and Preparation Method Thereof. CN n. 102038197 A, 2011. Disponível em: <http://p.espacenet.com/>. Acesso em: 13 maio 2013.

YOUSSEF, M. K.; BARBUT, S. Fat reduction in comminuted meat products - effects of beef fat, regular and pre-emulsified canola oil. Meat Science, Barking, v. 87, n. 4, p. 356-360, 2011. http:// dx.doi.org/10.1016/j.meatsci.2010.11.011

YUNES, J. F. F. Avaliação dos Efeitos da Adição de Óleos Vegetais como Substitutos de Gordura Animal em Mortadela. 2010. 103 f. Dissertação (Mestrado em Ciência e Tecnologia de Alimentos) - Centro de Ciências Rurais, Universidade Federal de Santa Maria, Santa Maria, 2010. 